\title{
LAS SITUACIONES PRÁCTICAS EN EL PROCESO DE ENSEÑANZA APRENDIZAJE DE LAS MATEMÁTICAS
}

\section{PRACTICAL SITUATIONS IN THE TEACHING-LEARNING PROCESS OF MATHEMATICS}

Agustín Márquez Oquendo, MSc.

framiya@dpe.pr.rimed.cu

Luis Enrique Hernández Amaro. Dr. C.

\section{RESUMEN}

Las situaciones prácticas constituyen una necesidad de la didáctica actual en la enseñanza preuniversitaria, sobre todo, porque mediante las cuales se puede dirigir el proceso de enseñanza aprendizaje, de manera que resulte un aprendizaje duradero, significativo y de interés para los alumnos. Está sustentada en la teoría de las situaciones didácticas de Guy Brousseau y sus seguidores, el aprendizaje desarrollador de los seguidores cubanos de Majmutov y Vigotsky, la teoría marxista del conocimiento, y otras, lo cual unido a su definición se encuentran reseñados en este artículo.

Palabras clave: situaciones prácticas, tratamiento de situaciones prácticas, situaciones didácticas, enseñanza aprendizaje desarrolladores

\section{ABSTRACT}

Practical situations constitute a necessity on current didactics mainly at pre-university teaching, especially because through them, the teaching learning process can be directed, so that it becomes a durable, significant learning of interest for the students. This study is based on the theory of didactic situations of Guy Brousseau's and followers, the learning development theory of Majmutov and Vigotsky's Cuban followers, the Marxist theory of knowledge, and others, which together with their definition are pointed out in this article.

Key words: practical situations, treatment of practical situations, didactic situations, teaching-learning development.

Recibido: febrero de 2016 Aprobado: abril de 2016

\section{Introducción}

Las situaciones prácticas en el proceso de enseñanza deben verse como un ente didáctico, como un medio que propicia el conocimiento de manera activa y que permite su más estrecha vinculación con la vida en el entorno donde se inserta el alumno.

Al concebir la clase como un espacio donde se produzcan conocimientos, implica tomar posición con respecto al aprendizaje, apareando en el mayor grado posible el conocimiento que se obtiene en la escuela y el que se produce fuera de ella.

Uno de los precursores en la concepción de la clase como ámbito de producción de conocimientos a través de situaciones didácticas, fue Guy Brousseau desde 1986, específicamente para las Matemáticas, quien basa sus postulados en preceptos del constructivismo genético de Piaget, pero si se toman los postulados de la escuela histórico cultural de Vygotsky, esta concepción toma una riqueza mayor, dadas las posiciones filosóficas asumidas en esta tesis, y por el amplio espectro que abarca dicha escuela, la cual tiene seguidores que aportan otros elementos que los contextualizan a la realidad educativa en Cuba, aún cuando Piaget toma en cuenta la herencia cultural.

Como se puede constatar, esta concepción es válida, a juicio del autor, para todas las asignaturas, y toma una connotación mayor en la enseñanza preuniversitaria.

La contradicción entre los anteriores y los nuevos conocimientos son la fuente indispensable para 
la producción de los nuevos saberes, proceso que en la enseñanza preuniversitaria tiene un estadio especial por la madurez de los alumnos con relación a los grados anteriores, por las características personales que van alcanzando: ya tienen criterios propios, son menos propensos a las imposiciones y sienten la necesidad de sentirse protagonistas de sus propias acciones, de ser escuchados, por lo que su participación en cualquier actividad debe ser asesorada, orientadora, de apoyo, no impuesta.

Para que el alumno sienta la necesidad de enfrentar una tarea cualquiera debe sentirse motivado hacia esta, para lo cual necesita tener los recursos cognitivos y procedimentales básicos para enfrentarla, conocer además, la utilidad, la trascendencia que tiene en su formación, en su vida personal, hacia esta dirección se encamina el presente artículo.

\section{Desarrollo}

Para acceder al conocimiento se necesitan medios que permitan insertar las relaciones que se producen en el proceso de solución de la situación práctica. Dichos medios deben estar en manos del alumno antes de que se enfrente a la "producción del conocimiento", de modo que se convierta en un protagonista, con el profesor de moderador de la actividad.

En la enseñanza preuniversitaria, es necesaria la realización de una sistematización y profundización de los conocimientos que tienen los alumnos, relacionados con el contenido de la asignatura y los procedimientos de trabajo, lo que les permitiría una actitud positiva ante la gestión del conocimiento y su ampliación.

No se trata de que el alumno sea quien exactamente produzca solo el conocimiento, sino de que tenga la mayor participación, por lo que la intencionalidad didáctica debe estar presente en la envoltura lingüística de la situación práctica y la preparación del grupo para enfrentarla, buscando la mayor aproximación a la forma que en la práctica se le pueda presentar. Estos preceptos son aplicables en la enseñanza a través de situaciones prácticas, en la que llega un momento en que la intervención del profesor es necesaria para aportar la parte del conocimiento que el alumno no puede "descubrir" y por tanto organizarlo en un sistema, en el que la teoría se ha heredado, construido y fundamentado, culturalmente.

Según la teoría de Brousseau (1986) y sus seguidores, la situación didáctica contiene varios aspectos, los cuales son extensibles a las situa- ciones prácticas, las cuales, además, contemplan el aspecto axiológico, significativo, que tiene trascendencia en la vida actual y posterior del alumno, por tanto estas tienen sus particularidades.

El autor considera que deben tenerse en cuenta las dimensiones del aprendizaje desarrollador, pues amplían aún más el espectro de acción de las enunciadas por la Teoría de las Situaciones Didácticas. Llevadas a situaciones prácticas las hacen situaciones específicas de aprendizaje en casi todas las asignaturas del currículo en preuniversitario: (Anexo \# 1 Esquema \#1, Componentes de la enseñanza aprendizaje desarrolladores aplicados a las situaciones prácticas)

Los indicadores son propios de cada asignatura adaptándose a las características de cada una.

En la activación-regulación, se dan los elementos a tener en cuenta, más estrechamente relacionados con el aspecto de la producción del contenido, contempla los aspectos con mayores insuficiencias en el aprendizaje de las matemáticas, sobre todo en lo que a creatividad se refiere.

En la significatividad. Como la denominación de la dimensión indica, se refiere a los aspectos relacionados con lo que puede hacer que el alumno quiera aprender, el valor que le encuentra al contenido, a la actividad misma de producción del contenido.

La motivación refiere lo relacionado con aquellos aspectos que desde el punto de vista sicológico, afectivo, volitivo, axiológico se producen en el alumno desde y hacia el aprendizaje.

La relación del aprendizaje desarrollador con la situación práctica, propicia la eliminación de una de las constricciones que se produce en determinados casos cuando el docente se hace creer y hace creer al alumno que produjo un conocimiento, cuando lo que hizo fue reproducirlo o responder de algún lugar lo que ya está elaborado según una guía o una sucesión de pasos algorítmicos, dando lugar a un fraude epistemológico (D'Amore, 2008), cuyo esencia tiene el profesor, preconcebida. Dicha condición es del tipo didáctico, que termina con oprimir el desarrollo cognitivo, creativo, la autogestión productiva.

La envoltura lingüística de la situación práctica, el clima que se crea para garantizar el enfrentamiento a la misma y la preparación del alumno con recursos que le permitan asumir un papel protagónico, son esenciales para, en determinados momentos, cuando el contenido mismo sea factible, propiciar una situación práctica adidáctica, de la cual el profesor puede obtener una aproxima- 
ción cada vez mayor del nivel que van alcanzando los alumnos en el proceso creativo, y acercarlos más, con un pensamiento activo, a la producción de conocimientos en clases.

En la envoltura lingüística debe estar explícito el entorno en el que produce la situación y conminar al alumno a enfrentarla, pero no de manera didactizada extrema, en el que no le resulte de ninguna dificultad al alumno saber hacia dónde dirigir su atención, más debe resultar de la inquietud que cause en él, el planteamiento de dicha situación.

Como su nombre indica, la situación práctica es el traslado al salón de clases de una cuestión real a la cual se le va a dar un tratamiento con herramientas propias de la asignatura que se trate, de modo que quede resuelta y que deje en el resolutor una huella en los modos de actuación cuando ingrese en la universidad si es el caso, o cuando ya no esté en la escuela y tenga que enfrentar la sociedad como un ser activo, protagónico en ella, convertirse en un difusor de cultura en la comunidad, en la familia, en el entorno de sus amistades y se puedan establecer debates con conocimiento de causa, no digerir las informaciones como se las presentan exactamente, sino que sea capaz de motivarse por conocer más, por profundizar en el tema y buscar por sí mismo o con ayuda de otros, otras situaciones derivadas de la primera.

Cuando se está trabajando con situaciones prácticas, el desconcierto en el docente e inicialmente en parte de los alumnos está latente, pues se produce un momento de búsqueda de estrategias, de organización y conexión de ideas, de búsqueda de vías, de recursos en general, y ahí precisamente está el hecho de mayor valor, es algo que se debe potenciar, no es pérdida de tiempo que los resultados no se obtengan de inmediato, pues una vez que se obtienen, son más duraderos según ha podido constar el autor a través de su experiencia empírica y la consulta de materiales científicos relacionados con ello (Brousseau, 1999; Zilberstein, 1999; Torres,1999; Silvestre y Zilberstein, 2000; Galindo, 2007; Sadovsky, 2013; entre otros).

El cotejo y la discusión de los resultados son elementos a tomar en consideración por la importancia que se le da a la producción del alumno, momento en que se siente tomado en cuenta y que su actividad es valorada. En este momento el profesor necesita dominio suficiente, no solo del contenido de la asignatura, sino de la maestría pedagógica, para lograr aprovechar todo el valor de los resultados obtenidos por los alumnos y desestimar los que se alejaron de la verdad. El criterio definitivo no debe ser impuesto, sino con- ciliado, y el autor considera que esta es una parte del contrato didáctico que se alude muy poco en la literatura.

Es importante la evaluación en tiempo real que se haga de la actividad, de los resultados obtenidos, permite detectar quiénes tienen mayores dificultades en el proceso de producción, de creación, de establecimiento de relaciones y conexiones, y que por tanto necesitan de un entrenamiento mayor, de niveles de ayuda acordes con sus limitaciones. Permite focalizar los que mayores posibilidades tienen y por tanto, diferenciar los niveles de dificultad de las tareas docentes que se orienten.

El entrenamiento de los alumnos en la forma de trabajo, de enfocar las situaciones y lograr la respuesta adecuada, debe ser sistemático, enfrentando a los alumnos a situaciones extremas en las cuales, llegar al resultado no está explícitamente declarado, sino que se impone por la lógica de la respuesta, de la respuesta a las exigencias que se debe obtener, por la práctica misma de la situación.

El entrenamiento sistemático de los alumnos para enfrentarse a situaciones prácticas y producir conocimientos, obtener resultados adecuados, es avanzado cuando se proponen situaciones, no solo dimensionadas, sino subdimensionadas, sobredimensionadas e imposibles. Incluso resulta altamente productivo, como ha podido constatar el autor en el aula, y ha podido obtener de otros autores (Schoenfeld, 1987; D'Amore, 1993; D'Amore-Martini, 1997), siempre que sea posible enfocar una misma cuestión desde diferentes aristas, de modo que se pueda mostrar la necesidad de analizar, de llegar a fondo en la respuesta, en el resultado, y no considerar lo que el profesor pueda esperar o pensar, lo que el alumno mismo está, tradicionalmente, acostumbrado a hacer.

Un aspecto esencial resulta entender que no es lo mismo el conocimiento científico, sistematizado, estructurado, heredado culturalmente, producido por una comunidad de expertos, que el conocimiento académico, escolar, que debe producirse en el aula como parte de ese contenido científico, que se necesita para comprender la realidad en determinados aspectos, según los objetivos de un programa concebido intencionalmente y las características del grado, siguiendo reglas y principios inviolables.

La necesidad de mover el pensamiento de los alumnos, más bien de removerlo, teniendo en cuenta la forma tradicional en que se ha establecido el contrato didáctico entre profesores y alumnos en las enseñanzas anteriores, constituye una 
necesidad impostergable, por cuanto el preuniversitario es la antesala de la universidad, donde el rigor de la enseñanza aprendizaje exige un nivel mayor de independencia y gestión del conocimiento por parte del alumno, entre otros aspectos.

El tratamiento de las situaciones prácticas, su inserción en las clases de Matemáticas

El tratamiento de las situaciones prácticas en las clases de Matemáticas se refiere a la forma en que las cuestiones de la vida, la ciencia, la práctica en general, que precisan de procedimientos matemáticos, se abordan en las clases, desde la preparación para resolverlas, formularlas, hasta la aplicación que se les da en diferentes contextos, todo el proceso en el cual se trabaja con estas, el conocimiento de los aspectos que las componen, sus características, formas de enfrentarlas en el proceso de solución y formulación, el uso posterior que deben tener en la vida de cada uno de los alumnos, al clima que se debe propiciar para que sean creíbles, aceptadas y asimiladas por la inmensa mayoría de los alumnos.

Las situaciones prácticas tienen sus antecedentes, por tanto, en las situaciones didácticas, la enseñanza problémica, y el aprendizaje desarrollador, con características generales que las convierten en un ente más amplio, acorde con las exigencias actuales de la educación en Cuba, particularmente en lo relacionado con la educación matemática.

Tal definición, situación práctica, debe contener la esencia de los objetivos que se persiguen con su tratamiento y los resultados que se esperan de ella, además las otras características que la identifican.

Una situación práctica es una determinada cuestión de la vida que es necesario resolver, y requiere, por parte de quien la enfrenta, de un conocimiento del tema que trata, del campo en el que se mueve, para poder justificar los pasos a ejecutar y poder aseverar la acepción o no de la respuesta, de acuerdo a los resultados obtenidos y la exigencia que plantea, cuya vía puede ser desconocida o no en dependencia del nivel de preparación del resolutor, generalmente encierran un valor axiológico y sus resultados tienen aplicación en otros contextos.

Las situaciones se dan en la realidad, existen siempre, los cuestionamientos de estas se alumbran cuando surge la necesidad de resolver algún aspecto de dicha situación y la solución no es inminente. Cuando se convierten en una necesidad las asumimos como un reto personal y decidimos en consecuencia dedicarle tiempo y esfuerzos para procurar resolverlas desconociendo la vía.

Las situaciones prácticas toman una dosis de problemicidad mayor cuando, desde el punto de vista procesal, no se evidencia la vía que la resuelve, pero con la aplicación creadora de conocimientos se puede llegar a ella, o con el trabajo colectivo se puede construir.

Las situaciones prácticas constituyen, entonces un ente que se distingue por la forma en que se le presentan al alumno, la forma que se tratan en las clases de Matemáticas, de modo que no siempre tiene que ser una situación que demande un procedimiento de solución desconocido o no inmediato, sino que el objetivo esencial es asegurar que le llegue al alumno, que este vea su aplicación en la vida y sienta ganas de enfrentarla, que pueda vincular contenidos matemáticos y obtener una enseñanza vivencial, cada vez más expedita de aquellas cuestiones que se le presenten en la vida.

Evita el rechazo que hacen docentes y alumnos a los problemas, como un concepto más general, algo que recordarán de alguna forma muchos años después de haber concluido los estudios, y permite que los profesores lo asuman como un nuevo tipo de ejercicio, que en realidad les sirve para resolver cuestiones prácticas y profundizar en los conocimientos matemáticos, con sus particularidades que lo hacen más digeribles y permiten una predisposición más favorable. Tal definición contiene la esencia de los objetivos que se persiguen con su tratamiento y los resultados que se esperan de ella, además las otras características que la identifican.

Las situaciones prácticas en Matemáticas, acercan más el contenido a los aspectos relacionados con el plano axiológico y de significatividad del aprendizaje.

En la clasificación de ejercicios se adecua a los ejercicios de nuevo tipo (ENT) según la concepción de Ballester (1995), aunque concebidas no solo para la sistematización de los conocimientos, sino también para su producción.

Los preceptos planteados por Ballester (1995) se adecuan sin dificultad a las situaciones prácticas, solo que en estas se refuerzan el aspecto axiológico y motivacional como parte de la sistematización y producción del conocimiento, para lograr que se fije a mayor plazo.

Cuando se habla del tratamiento de situaciones prácticas en las clases de Matemáticas, sea cual sea la enseñanza hay que diferenciar dos fines: 
- La práctica contextualizada en el uso de las herramientas matemáticas que se están tratando.

- La aplicación práctica de las matemáticas en la vida del alumno desde diferentes aristas: axiológica, práctica y afectivo-volitiva.

En el primero de los casos no se justifica la didactización extrema del texto de la situación práctica, de modo que funcione como un comando meramente formal, una orden de ejercicio que le indique al alumno lo que debe enfrentar de manera directa, lo que implica no obviar la ilustración real de lo que puede utilizar en la práctica, o sea se plantea como una situación adidáctica según lo tratado en el epígrafe anterior.

El segundo caso es más complejo, pues implica poner al estudiante en función de resolver una situación que se le puede presentar de la manera que se propone o una muy similar en la vida práctica, lo cual implica que a través de una serie de razonamientos, el alumno se vea en posición de investigar, de enfrentar la situación, de descubrir el proceder para enfrentarlo dentro y fuera del aula, una situación en la cual el proceder matemático y las relaciones matemáticas no son dados de manera evidente, sino que requieren de la abstracción del sujeto, de la interpretación de la situación para obtener las relaciones matemáticas, que pueda descubrir las relaciones matemáticas en las propiedades y/o características de los objetos en la vida, surgida de la propia exigencia planteada.

Su uso es más amplio, toda vez que no siempre tiene que implicar una vía desconocida, lleva una mayor carga de función axiológica, práctica, afectivo-volitiva, de independencia, y significado para el alumno, además de la preparación teórica que recibió que le sigue siendo de utilidad en la vida. Puede palpar la aplicación de los resultados que ha ido obteniendo en diferentes contextos, que desde el aula se anunciaron. Se entrena al alumno en su uso.

Si se toma en cuenta "La incapacidad para aplicar conceptos y modelos a situaciones dadas, de traducir un problema de la realidad a uno matemático. [8; p5], se debe pensar en la inminencia de acercar más la Matemática a las necesidades propias de los individuos que después deberán enfrentarse a la vida y que pocas veces les sirve de algo lo estudiado, ya que no lo recuerdan o no saben cómo relacionarlos.

Debe potenciarse el trabajo en las dos direcciones, con lo que el objetivo de las Matemáticas quedará cumplido lo más cercano a las aspiraciones concebidas en los programas de la asignatura y en los objetivos de la Educación en Cuba y el mundo. Las situaciones prácticas contienen tales ingredientes y su definición es más sugerente para tales propósitos.

En las múltiples situaciones que el individuo deberá enfrentar en la vida no siempre tendrá el conocimiento de la temática o tema en que se enmarcan las mismas, y tendrá que recurrir entonces, sin ayuda del maestro, a hurgar en las fuentes del conocimiento a través de diferentes vías y buscar, además, la manera de resolverlas.

Se asume que solo se trate del contenido, el alumno ya deba estar relacionado con la teoría de la resolución y formulación de situaciones prácticas, y estar en condiciones de poderla aplicar, ponerlo en condiciones de buscar el conocimiento matemático para enfrentar su solución o al menos plantearla.

Al alumno le es posible, con conocimientos de forma general, anteriores, llegar a comprender la situación práctica e iniciar el plan de ejecución, aún cuando las herramientas que obtenga no sean conocidas, ni mucho menos sus procedimientos de solución dominados, sino que haciendo uso adecuado de la orientación, para generar impulsos en los casos necesarios, se logre llegar al modelo, anteponiendo el clima que debe crearse para que el alumno se sumerja en el mundo en el entorno que da origen, pueda tener una base sobre las posibles estrategias a utilizar, el procedimiento de trabajo con la herramienta matemática, la forma en que debe dar la respuesta de acuerdo a las condiciones iniciales planteadas y se mueva en ese ambiente de modo que pueda valorar si va o no por el camino correcto.

Es en estos casos, como el mencionado, en el que el trabajo a través de situaciones prácticas es necesario para el tratamiento del contenido matemático con un significado evidente.

La situación que se aborda contiene determinados aspectos, que no limitan su uso en otros casos, y de hecho se pueda considerar de alcance general para el tratamiento de casos particulares, aún con las peculiaridades que puedan distinguirlas, incluso en otras asignaturas que tengan que hacer uso de este ente.

Es necesario conocer la esencia para entender el fenómeno, o viceversa según el caso [7; p.23] adaptando los preceptos de la enseñanza problémica y aprendizaje desarrollador a la solución de situaciones prácticas, más aún, con este enfoque 
podemos señalar entonces que en la situación práctica y todo el proceso que implica su solución "se refleja la contradicción dialéctica entre lo conocido y lo desconocido, entre el sujeto y el objeto del conocimiento, ... estimula la actividad cognoscitiva y desencadena todo el proceso de solución" de la misma. [13; p.2-3] También en la reformulación de lo conocido para extrapolarlo a otros casos, reorientarlo para obtener un nuevo conocimiento se produce una contradicción entre el estado inicial y el estado final del conocimiento a partir de los precedentes.

Generalmente, en la escuela se orientan "problemas matemáticos" solo de corte didáctico, es decir, los cuales generalmente el estudiante conoce de antemano la herramienta a utilizar, y tan explícito que prácticamente resulta muy evidente o no motiva al alumno, puesto que además se presentan de manera que en la vida no es como la encuentra. Se puede cumplir mejor la finalidad del trabajo con estos, cuando se enfrenta al alumno con la situación en la que se da el problema y se produce el conocimiento necesario, acercándola a como se la podría encontrar en la vida.

He ahí la oportunidad de la interrelación de materias, de la integración, no como suma de asignaturas en un mismo docente, sino la interrelación disciplinaria. El sujeto ha de conocer el tema, ubicarse en él, para entonces, en insuficiencias, a través de disquisiciones con las herramientas matemáticas, (en este caso) buscar la solución. $Y$ es que precisamente "el fundamento del conocimiento lo constituye el reflejo, que se caracteriza por ser psíquico y anticipado. Este reflejo de la realidad determina una importantísima función de la conciencia, la suposición del proyecto, precisamente sobre la base del reflejo adelantado de la realidad, el hombre se plantea los objetivos correspondientes, y conscientemente, subordina sus actos a ellos". [7; p.33].

El tratamiento de situaciones prácticas no consiste solamente en que los alumnos asimilen conocimientos acerca de los fenómenos, los objetos y los procesos de la realidad, sino en que asimilen los procedimientos de actividad que encierran los conocimientos, incluyendo la adquisición de experiencias en lo que respecta a la actividad creadora.

“.... únicamente la solución de las contradicciones que aparecen en el proceso de enseñanza (directamente durante el proceso de aprendizaje) puede constituir el proceso adecuado de asimilación creadora por el alumno, de dichos conocimientos y de los actos que con ellos se relacionan". [7; p.46] Lo cual es altamente productivo y de hecho se ha aprovechado en esta experiencia, para la obtención de definiciones, sobre todo de herramientas matemáticas y el procedimiento de trabajo con ellas a través de las mismas situaciones prácticas que las engendran.

\section{Conclusiones}

El tratamiento de las situaciones prácticas en las clases de Matemáticas se refiere a la forma en que las cuestiones de la vida, la ciencia, la práctica en general que precisan de procedimientos matemáticos se abordan en las clases, desde la preparación para resolverlas, formularlas, hasta la aplicación que se les da en diferentes contextos, esto se va concretando con la solución y/o planteamiento de situaciones prácticas, la formulación de hipótesis y la elaboración de preguntas deben provocar que en la búsqueda del conocimiento se entrene el pensamiento reflexivo, no solo la memoria, sino el razonamiento, la búsqueda de las causas, las relaciones y las consecuencias, la aplicación, reforzadas con el conocimiento que ha adquirido el alumno sobre los aspectos teóricos relacionados con las situaciones prácticas, profundizar en el tema que trata la situación, lo que posibilitará un aporte al volumen de conocimientos del alumno que bien le puede servir en la vida social, en el entorno donde se desenvuelva, o en el ámbito docente.

En este estudio, se valora al hombre, en tanto resuelve la mayoría de las tareas con ayuda de procedimientos que conoce. La actividad productiva incluye los procedimientos y métodos conocidos de la actividad mental, y al mismo tiempo crea un sistema nuevo de actos o descubre regularidades que antes eran desconocidas. Por esta razón, durante el proceso de la actividad productiva, el hombre descubre nuevas verdades también por la vía de las conclusiones; es decir mediante la demostración lógica y por la vía de la intuición (visión intelectual, sui géneris o incide, "destello" interno) es decir por una vía para la forma de concebir los ejercicios y darle tratamiento a la materia de enseñanza, al contenido, toda vez que se utilice la situación práctica como una vía de mostrar la necesidad de continuar profundizando en el conocimiento, la posibilidad de descubrir nuevos contenidos, de convertirse en un investigador novel.

Estos estudios contribuyen de manera decisiva al potenciamiento de los talentos matemáticos, la exploración constante de otros que todavía no se han hecho notar, e ir perfilando e incentivando las zonas de desarrollo próximo en los alumnos de menos capacidades mostradas. 


\section{Referencias Bibliográficas}

1. Ballester, Sergio Los ejercicios de nuevo tipo en la enseñanza de La Matemática, Dr. Sergio Ballester Pedroso Instituto Superior Pedagógico "Enrique José Varona", Artículos Colección Futuro, Eureka. (1995):

2. Brousseau G. Fondements et Méthodes de la Didactique des Mathématiques. Recherches en didactique des mathématiques. 7, 2, 33-115. (1986).

3. Brousseau G. Théorie des Situations Didactiques, Grenoble, La Pensée, Sauvage. (1998):

4. Brousseau G. Educación y didáctica de las Matemáticas. Educación Matemática. México. (1999):

5. D’Amore B. Epistemología, didáctica de la matemática y prácticas de enseñanza. Enseñanza de la matemática. Revista de la ASOVEMAT (Asociación Venezolana de Educación Matemática). Vol. $17, n^{\circ} 1,87-106$. (2008).

6. D'Amore B., Martini B. Contratto didattico, modelli mentali e modelli intuitivi nella risoluzione di problemi scolastici standard. La matematica e la sua didattica. 2, 150-175. En idioma español (1997): Números, 32, 26-32. (1997). En francés : Scientia Paedagogica Experimentalis, XXXV, 1, 95-118. En inglés en: Gagatsis A. (editor) (1999). A multidimensional approach to learning in mathematics and science. Nicosia: Intercollege. 3-24.

7. Majmutov, M.I. "La enseñanza problémica", Editorial Pueblo y Educación, La Habana. (1983):

8. MINED Seminario Nacional para Educadores. Tabloide. Ministerio de Educación. (2004):

9. Pogglioll, L. "Perspectivas actuales de la Investigación en el área cognoscitiva". Instituto Pedagógico de Caracas, INTERNET. (2001):

10. Ron Galindo, José.“Una estrategia didáctica para el proceso de enseñanza-aprendizaje de la resolución de problemas en las clases de matemática en la educación secundaria básica". Tesis presentada en opción al grado científico de Doctor en Ciencias Pedagógicas. Ciudad de La Habana. (2007):

11. Sadovsky, Patricia: "La Teoría de las Situaciones Didácticas: un marco para pensar y actuar en la enseñanza de la Matemática". http://www. matematicassinaloa.com/Informacion/ /Documentos/26_La\%20Teoria\%20de\%20Situaciones\%20
Didacticas.pdf

12. Schoenfeld A.H. What's all the fuss about metacognition? En: Schoenfeld A.H. (editor) (1987b). Cognitive science and mathematics education. Hillsdale (N.J.): Lawrence Erlbaum Ass. 189-215. (1987).

13. Silvestre, M. y J. Zilberstein: "Enseñanza y aprendizaje desarrollador", Ediciones CEIDE, México. 2000

14. Silvestre, M. y J. Zilberstein "Enseñanza y aprendizaje desarrollador", Ediciones CEIDE, México. (2000):

15. Torres Fernández, Paúl. Didácticas cubanas en la enseñanza de la Matemática. Enseñanza de la Matemática a partir de una célula generadora. Proposiciones metodológicas. Editorial Academia. La Habana. 1999.

16. Zilberstein, J., J.R. Portela y M. Macpherson:: "Didáctica integradora de las Ciencias vs Didáctica Tradicional, Experiencia Cubana", Editorial Academia, C. Habana. 1999. 
Anexo \# 1

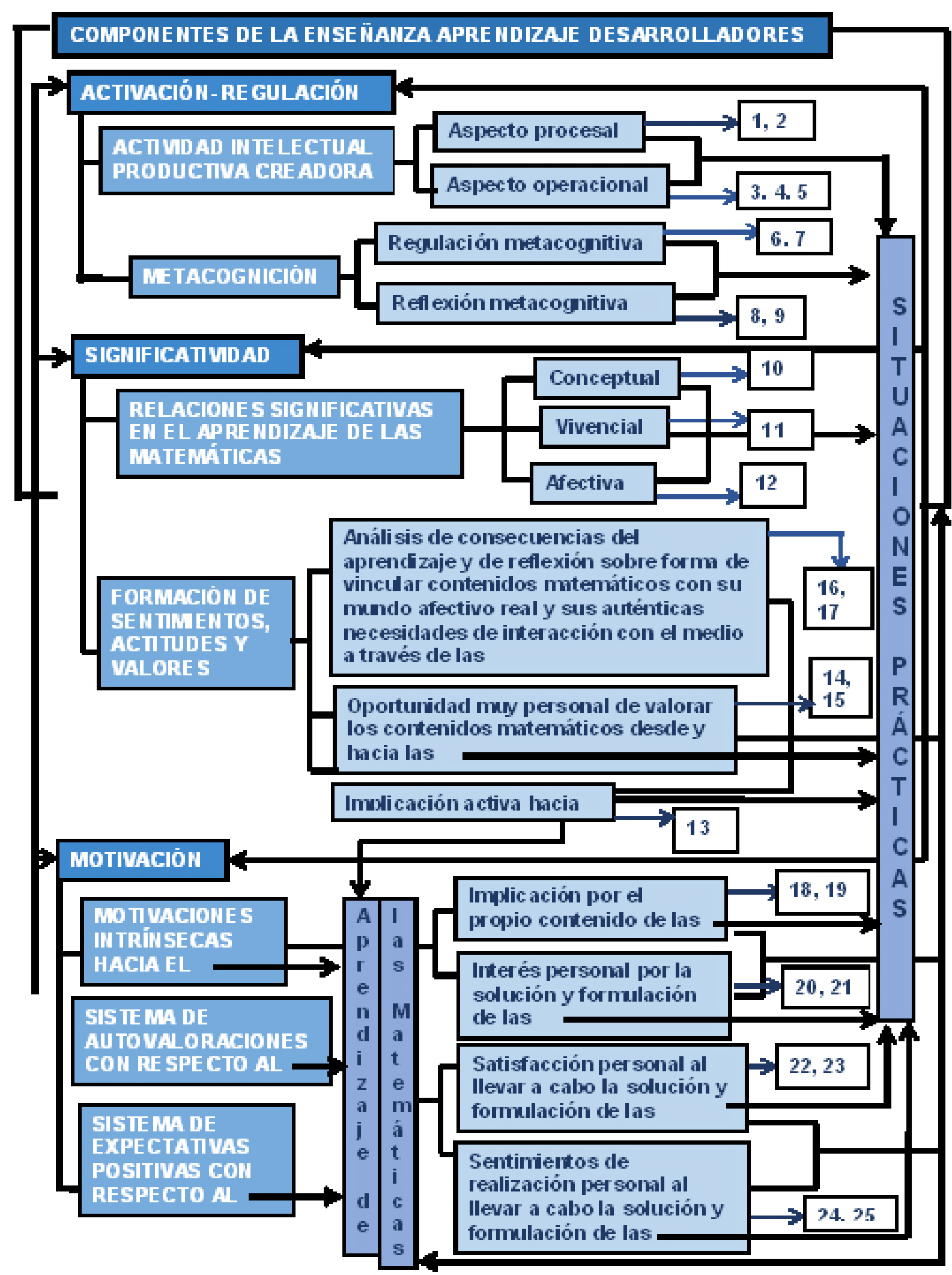

Esquema \#1. Componentes de la enseñanza aprendizaje desarrolladores aplicados a las situaciones prácticas 